\title{
Integrated practical classes in photonics at the Vrije Universiteit Brussel
}

Kristel Praet, Hugo Thienpont

Kristel K. Praet, Hugo Thienpont, "Integrated practical classes in photonics at the Vrije Universiteit Brussel," Proc. SPIE 3190, Fifth International Topical Meeting on Education and Training in Optics, (8 December 1997); doi: $10.1117 / 12.294401$ 1997, Delft, Netherlands 
Integrated Practical Classes in Photonics at the Vrije Universiteit Brussel

Kristel Praet and Hugo Thienpont

Vrije Universiteit Brussel, Lab for Photonic Computing and Perception, Pleinlaan 2, B- 1050 Brussel, Belgium

e-mail: kpraet@vub.ac.be

\begin{abstract}
Photonic technology definitely plays an important role in the worlds of informatics, telecommunications and instrumentation. Therefore, at the faculty of applied sciences of the Vrije Universiteit Brussel, we have established a new multidisciplinary engineering curriculum in Photonics, where besides theoretical courses, a lot of time is spent giving students a hands-on, in depth practical training. In this paper we focus on the different innovative aspects of our approach to train basic and practical photonic skills of EE students.

The concept of the practical classes is special in the sense that they encompass the different theoretical courses, like "Diffractive and Fourier Optics", "Physical properties of optical materials and artificial structures", "Optical telecommunication", "Displays" and "Computer aided design of optical and opto-mechanical systems".

Students acquire the methodology and the skills to work with modern, sophisticated instrumentation and experience team work.

In a first phase, the integrated practical classes are concerned with teaching students how to make basic table-top set-ups with modular opto- mechanical elements, using visible lasers and opto-electronic devices. In addition, they learn how to use measuring and diagnostic equipment and how to interpret measurement results.

The next step consists in dealing with mini-projects. Topics can be either academically or industrially oriented. Here we focus on "How to report, communicate and disseminate results".

We strongly believe in combining the assimilation of fundamental concepts with the acquisition of practical skills in electronic and photonic instrumentation, design methods and experimental set-ups to form flexible and practically inspired photonic engineers.
\end{abstract}

Photonics, training, integrated practical classes

\title{
KEYWORDLIST
}

\section{INTRODUCTION}

A new era for optics really started in 1960 with the production of coherent light by a laser. Moreover there was the combination of laser light with fiber optics and charged coupled devices a technological breakthrough at that time. In the 1970 's the word photonics arose to describe the use of photons in ways analogous to the use of electrons and the field of optical communication was being explored. Known examples for the 1970's are images coming from satellites, light guiding of laser beams, holography, spectroscopy, biomedical applications and the integration of optical components on integrated circuits. With the 80's the optical industry started mass production of lasers and detectors for use in printers, fax machines and $\mathrm{CD}$ players. With new light sources for optical communication, innovative optical fiber technology, optical biomedical applications and machine vision, the optics-electronics industry was experiencing a boom.

Because of these rapid evolution's and huge progresses of optical techniques, materials and devices and the increasing relationship between electronics and optics, there was a need to define this new field of photonics, that binds all these related technologies, merging electronics and applied physics.

In a broad sense photonics could be described as the generation, manipulation, transport, detection and use of light information and/or light energy, where light refers to electromagnetic radiation from the far infrared to the x-ray region.

In those applications where electronic circuits and components are limited, for example concerning bandwidth and interconnection capacity, light and photons can take over. 
The background of this emerging domain is essentially the electromagnetic theory, the conventional optics, wave and photon optics, theory of interaction of light and matter and the semiconductor theory. These theories form the roots, to fully understand the sub-disciplines of photonics like for examples guided-wave and fiber optics, electro-optics, optical communications and optical computing and switching. The basic tools used are light sources, different optical components and detectors whereas the basic principles used are modulation, switching, scanning, amplification and detection.

Photonic technology now definitely plays an important role in the worlds of informatics, telecommunications and instrumentation.

Photonics and its applications are growing so rapidly, that an appropriate training in this field is a necessity. Therefore, at the faculty of applied sciences of the Vrije Universiteit Brussel, we have established a new multidisciplinary engineering curriculum in Photonics, where besides theoretical courses, a lot of time is spent giving students a hands-on, in-depth practical training. In this paper we focus on the different innovative aspects of our approach to train basic and practical photonic skills of EE students, during the eighth, ninth and tenth semester of their study program.

\section{INTEGRATED PRACTICAL CLASSES IN THE PHOTONICS CURRICULUM}

\subsection{Introduction}

The concept of the integrated practical classes is special in the sense that they encompass the different theoretical courses, like "Diffractive and Fourier Optics", "Physical properties of optical materials and artificial structures", "Optical telecommunication", "Displays" and "Computer aided design of optical and opto-mechanical systems". These integrated classes are not just demonstrations of the different parts taught in one course but fully exploit the ability of the students to actually link specific theories and handle multidisciplinary problems.

We strongly believe in combining the assimilation of fundamental concepts with the acquisition of practical skills in electronic and photonic instrumentation, design methods and experimental set-ups to form flexible and practically inspired photonic engineers.

The concept of the classes is carried out such as to promote skills that reach further than scientific knowledge: initiative and creativity, flexibility but also decision making and professionalism are stimulated in an environment where communication, languages and team work play a key role. The students reach a very high level of scientific knowledge combined with the very important behavioral aptitudes they need to function in an optimal way in their future work environment. ${ }^{1}$

\subsection{Concept of the classes}

Over the two specialization years for photonic engineers, a total of 120 college hours is spent on the practical classes.

In addition mini-projects have to be carried out followed by a diploma work.

In the eighth semester students have to accomplish 5 case studies in teams of two, three or four students. The approach of these case studies is special in that way that we don't believe in giving these students the recipes of what they should do in the lab. They receive information and documentation concerning the case study together with some well defined goals of the project, involving both theory and practical work.

From these instructions they can prepare, look into manuals and course notes for the principles, formulas and background they need to accomplish the study. They come to the lab with these preparations and seek for themselves the mechanical and electronic equipment they need and start setting up, by trying, making mistakes and iteration processes.

With this procedure they have the possibility to learn for themselves how to build set-ups and therefore their curiosity and creativity is greatly stimulated and the challenge for them is enormous.

Students acquire the methodology and the skills to work with modern, sophisticated an expensive instrumentation, they learn to contact companies select material in catalogs, compare prices and experience team work.

Also during this semester they finish a mini diploma work, academically or industrially oriented, like for example the installation of an optical sensor in a dam or medical image processing techniques.

The approach for the classes in the two following semesters is again different since the emphasis here is laid on handling problems, projects or systems with a focus on "How to report, communicate and disseminate results".

Again in groups they tackle problems using the knowledge and experience they have built up the former year.

We also ask them to write up their work in the format of a scientific paper (in English) and present it as it were a conference talk, which prepares them fully for the public defense of their diploma work.

Diploma works can be purely academic, for example the study of the polarization behavior of Vertical Cavity Surface Emitting Lasers, or real engineering work in cooperation with industry, like the study and realization of a laserprinter, or they can tackle a multidisciplinary topic, like polarimetric optical fiber sensors and tension analysis for medical applications. Very important contacts, projects and cooperation with foreign universities have been established through and for diploma works in the framework of Erasmus, Socrates and Tempus program. 


\section{INTEGRATED PRACTICAL CLASSES}

The integrated practical classes of the eighth semester are concerned with teaching students how to make basic table-top setups with modular opto-mechanical elements, using visible and infrared lasers and opto-electronic devices. In addition, they learn how to use measuring and diagnostic equipment and how to interpret measurement results.

Case studies can be the characterization of visible Helium neon lasers or infrared diode lasers, where emphasis is laid on measurements of intensity distribution, beam waist, divergence, beam profile and total power. Here different methods and equipment can be used. Other examples are the characterization of a liquid crystal retarder, the study of different types of optical fibers and holograms.

In the ninth semester they tackle mini projects like for example the realization of an optical fiber interferometric sensor or building a two channel WDM system, including transmitter and receiver electronics. Herefore they use the acquired knowledge about theory, experiments and instrumentation to handle projects with a professional approach. Semester ten is devoted to the diploma work.

\section{CONCLUSIONS}

We believe that this way of learning practical skills is extremely motivation and contributes to the students' personality and professionalism. The important goals for the integrated practical classes in photonics are the students' personal achievements in the realization of set-ups and experiments, the flavour to work with sophisticated and expensive instrumentation and acquiring interpretation and diagnostic skills. Aspects of foreign languages, team work and oral and written communication skills have also proven to be motivating and prepares students in a professional way to their future work environment.

Our photonics program was developed with a limited budget since no special funding was available for transforming the training discipline. We only had at our disposal a small budget that was left from services rendered to industry. Notwithstanding this, fascinated by the unique opportunity we were facing, we took advantage of the small-size and the flexibility of our faculty to create this photonics discipline according to one of the principles of the "Learning Society", as defined by Sir Christopher Ball: " Learning is a partnership between students, teachers, parents, employers, and the community working together." ${ }^{2}$

\section{REFERENCES}

1. I. Veretennicoff and H. Thienpont, "Teaching optics for other disciplines”, Invited Paper at the fifth International Meeting on education and Training in optics, Delft, SPIE Proceedings vol. 3190, August 19-21 (1997). 2. H. Thienpont and I. Veretennicoff, "Photonics in Brussels: Innovation in Electrical Engineering Education.", Optics \& Photonics News, p. 52-53, April 1996. 